\title{
An examination of the Clinical Impairment Assessment among women at high risk for eating disorder onset
}

Denise E. Wilfley

Anna Vannucci

Andrea E. Kass

Meghan M. Sinton

William \& Mary, mmsinton@wm.edu

\section{Recommended Citation}

Vannucci, A., Kass, A. E., Sinton, M. M., Aspen, V., Weisman, H., Bailey, J. O., ... \& Taylor, C. B. (2012). An examination of the Clinical Impairment Assessment among women at high risk for eating disorder onset. Behaviour research and therapy, 50(6), 407-414.

This Article is brought to you for free and open access by the Arts and Sciences at W\&M ScholarWorks. It has been accepted for inclusion in Arts \& Sciences Articles by an authorized administrator of W\&M ScholarWorks. For more information, please contact scholarworks@wm.edu. 


\title{
An examination of the Clinical Impairment Assessment among women at high risk for eating disorder onset
}

\author{
Anna Vannucci ${ }^{\mathrm{a}}$, Andrea E. Kass ${ }^{\mathrm{b}}$, Meghan M. Sinton ${ }^{\mathrm{c}}$, Vandana Aspen ${ }^{\mathrm{d}}$, Hannah Weisman ${ }^{\mathrm{d}}$, \\ Jakki O. Bailey ${ }^{\mathrm{d}}$, Denise E. Wilfley ${ }^{\mathrm{b}, \mathrm{e}, *}$, C. Barr Taylor ${ }^{\mathrm{d}}$ \\ ${ }^{a}$ Uniformed Services University of the Health Sciences, Department of Medical and Clinical Psychology, 4301 Jones Bridge Road, Bethesda, MD 20815, USA \\ ${ }^{\mathrm{b}}$ Washington University in St. Louis, Department of Psychology, St. Louis, MO 63110, USA \\ ${ }^{\mathrm{c}}$ The College of William and Mary, Department of Psychology, Williamsburg, VA 23187, USA \\ ${ }^{\mathrm{d}}$ Stanford University School of Medicine, Department of Psychiatry, Stanford, CA 94304, USA \\ e Washington University School of Medicine, Department of Psychiatry, St. Louis, MO 63110, USA
}

\section{A R T I C L E I N F O}

\section{Article history:}

Received 5 November 2011

Received in revised form

22 February 2012

Accepted 22 February 2012

\section{Keywords:}

Eating disorders

Diagnosis

Clinical impairment

Psychometrics

Norms

Prevention

\begin{abstract}
A B S T R A C T
Identifying measures that reliably and validly assess clinical impairment has important implications for eating disorder (ED) diagnosis and treatment. The current study examined the psychometric properties of the Clinical Impairment Assessment (CIA) in women at high risk for ED onset. Participants were 543 women (20.6 \pm 2.0 years) who were classified into one of three ED categories: clinical ED, high risk for ED onset, and low risk control. Among high risk women, the CIA demonstrated high internal consistency $(\alpha=0.93)$ and good convergent validity with disordered eating attitudes $(r s=0.27-0.68, p s<0.001)$. Examination of the CIA's discriminant validity revealed that CIA global scores were highest among women with a clinical ED $(17.7 \pm 10.7)$ followed by high risk women $(10.6 \pm 8.5)$ and low risk controls $(3.0 \pm 3.3)$, respectively $(p<0.001)$. High risk women reporting behavioral indices of ED psychopathology (objective and/or subjective binge episodes, purging behaviors, driven exercise, and ED treatment history) had higher CIA global scores than those without such indices ( $p s<0.05$ ), suggesting good criterion validity. These data establish the first norms for the CIA in a United States sample. The CIA is psychometrically sound among high risk women, and heightened levels of impairment among these individuals as compared to low risk women verify the relevance of early intervention efforts.
\end{abstract}

(c) 2012 Elsevier Ltd. All rights reserved.
Central to the definition of a mental disorder is the notion of clinically significant distress and disability resulting from behavioral or psychological patterns (American Psychiatric Association [APA], 2000; APA, 2010). Disability refers to the experience of clinical impairment in one or more important domains of functioning, including occupational, academic, social, and role domains (APA, 2000; APA, 2010). Moving toward DSM-5, there is an increasing recognition that clinical impairment is a critical criterion to identify individuals in need of treatment because mental disorder symptoms may not always be associated with subjective emotional distress (Stein et al., 2010). The construct of clinical impairment may be especially relevant to determining the clinical significance of eating disorder psychopathology because many symptoms, such as intrusive thoughts related to an individual's shape and weight, can be ego-syntonic in nature (Polivy \& Herman,

* Corresponding author. Washington University School of Medicine, Department of Psychiatry, 660 South Euclid, Box 8134, St. Louis, MO 63110, USA. Tel.: +1 314286 2079; fax: +1 3142862091

E-mail address: wilfleyd@psychiatry.wustl.edu (D.E. Wilfley).
2002). Moreover, clinical impairment is often what prompts individuals with eating disorders to seek treatment and is considered a key treatment target and outcome measure (Fairburn, 2008). Therefore, the current study sought to examine clinical impairment among women with eating pathology.

Clinical impairment is a dimensional construct that varies greatly across a continuum (Stein et al., 2010). Therefore, research is needed to investigate clinical impairment in populations that vary by symptom severity to enhance diagnostic and treatment efforts. It is well established that full syndrome eating disorders are associated with marked clinical impairment as compared to individuals with low eating disorder psychopathology (Hudson, Hiripi, Pope, \& Kessler, 2007; Swanson, Crow, Le Grange, Swendsen, \& Merikangas, 2011). Preliminary evidence suggests that individuals with subclinical levels of disordered eating attitudes and behaviors may evidence psychological and medical consequences comparable to their full syndrome counterparts (Ackard, Fulkerson, \& NeumarkSztainer, 2011; Peebles, Hardy, Wilson, \& Lock, 2010). There has been no known empirical examination of the extent of clinical impairment in a population at high risk for eating disorder onset, 
such as those with elevated weight and shape concerns (Jacobi et al., 2011). An examination of clinical impairment across the broad range of eating pathology and eating disorder risk status would provide evidence for empirically distinguishing between clinically significant levels of impairment that indicate a need for treatment, moderate yet elevated levels of clinical impairment that identify high risk individuals and support the use of preventative interventions, and milder forms of clinical impairment that may not warrant intervention.

One criticism of the "clinical significance criterion" for mental disorders is that the constructs of distress and impairment are somewhat ambiguous and can rely too heavily on subjective judgments to determine clinical significance (Stein et al., 2010). The identification of psychometrically sound measures of clinical impairment would help to standardize and clearly operationalize this construct, thus bolstering researchers' and clinicians' confidence in the utility of the clinical significance criterion. The Clinical Impairment Assessment (CIA) was developed as a brief self-report questionnaire to assess the extent to which an individual's eating habits, exercising, or feelings about his or her shape, weight, or eating impact daily functioning in psychosocial domains (Bohn \& Fairburn, 2008). The CIA is distinguished from other eating disorder-related quality of life measures in that the CIA clearly emphasizes the severity of impairment across important domains of functioning that occurs as a direct consequence of an individual's eating disorder psychopathology, which has been suggested as a critical feature for determining clinical impairment in the DSM-5 (Stein et al., 2010). Thus, establishing the psychometric properties of the CIA in populations across a wide range of eating pathology and eating disorder risk has the potential for broader implications for clinical impairment assessment beyond the eating disorder field.

Previous studies have established the psychometric properties of the CIA in clinical and community samples, but none have occurred among a high risk sample. The initial study of the CIA occurred in women enrolled in an eating disorder treatment trial (Bohn et al., 2008). The CIA demonstrated excellent internal consistency and test-retest reliability in this sample (Bohn et al., 2008). The CIA was also strongly correlated with self-reported eating disorder psychopathology and clinician ratings of impairment at all time points throughout treatment, indicating good construct validity (Bohn et al., 2008). Finally, the CIA had adequate discriminant validity, such that a small sample of recovered patients $(n=37)$ reported significantly less impairment than those with full syndrome eating disorders $(n=33)$ (Bohn et al., 2008). A second study investigated the psychometric properties of the CIA in a community sample of young women with low eating pathology (Reas, Ro, Kapstad, \& Lask, 2010). Again, the CIA had excellent internal consistency and test-retest reliability as well as good construct validity with eating pathology (Reas et al., 2010). In the third study of the CIA, the measure was adapted to an interview format for use among adolescent schoolgirls with clinical and subclinical eating disorder symptoms from rural Fiji (Becker et al., 2010). The CIA interview format was tested among 215 schoolgirls and found to be internally consistent and to have adequate criterion and construct validity with measures of eating disorder psychopathology (Becker et al., 2010). Taken together, these results suggest that the CIA is a reliable measure in young women with full syndrome eating disorders and community samples. The CIA has also consistently demonstrated construct validity in previous studies; however, there has been limited work on the criterion and discriminant validity. An important next step in determining the clinical utility of the CIA is to examine its psychometric properties among individuals at high risk for eating disorder onset.

The establishment of norms for clinical impairment measures such as the CIA provides a useful framework within which clinicians can interpret varying levels of impairment severity. Normative data may allow the CIA to serve as a potentially powerful tool to estimate symptom severity and supplement clinicians' decisions regarding treatment planning. Mean CIA global scores in prior studies ranged between 6 and 9 in healthy women from Sweden and Norway and in adolescents from Fiji (Becker et al., 2010; Reas et al., 2010; Welch, Birgegard, Parling, \& Ghaderi, 2011). On the other hand, a mean CIA global score of approximately 30 has been estimated for those diagnosed with eating disorders in clinical samples from the United Kingdom and Sweden (Bohn et al., 2008; Welch et al., 2011). To date, there have been no known studies of the CIA within the United States. Due to potential cultural differences, it is unclear as to whether the CIA norms will be the same as in previous studies; thus normative United States data for the CIA is important to inform the generalizability of study findings.

The primary objective of the current study was to examine the psychometric properties of the CIA for a sample of college-age women at high risk for developing an eating disorder. We hypothesized that the CIA would demonstrate excellent internal consistency and good criterion and convergent validity in relation to eating pathology among high risk women. We also expected to replicate the CIA's factor structure. In terms of discriminant validity, we further anticipated that CIA global scores would increase as risk level increased from low risk, high risk, to clinical eating disorder groups. As a secondary objective, we sought to extend previous studies by establishing norms across the spectrum of disordered eating in sample from the United States and to compare these norms with results from prior studies.

\section{Methods}

\section{Participants}

Participants were women at varying levels of eating disorder risk between 18 and 25 years of age and had a body mass index between 18 and $32 \mathrm{~kg} / \mathrm{m}^{2}$. Women were recruited broadly from two private universities and three public colleges/universities in the Sacramento and San Francisco Bay areas and from one community college, one public university, four small graduate schools/liberal arts colleges, and three private universities in the Saint Louis metropolitan area. The vast majority of these women were enrolled in undergraduate or graduate level courses at these local universities and colleges. Interested women were excluded if they were actively suicidal or psychotic, were suffering from bipolar disorder, did not have regular Internet access, or resided outside the metropolitan regions of the university sites. Women who reported current prescription medication for mood or anxiety disorders were included if their medication was stable for at least two weeks.

\section{Procedures}

Study participants were recruited via study fliers posted at local academic institutions, Facebook, Craigslist, campus email solicitations from study staff and campus leaders, and a recruitment organization called Volunteers for Health (only at Washington University). Recruitment materials were broadly targeted for women who were concerned about their weight, wanting to feel better about their body, experiencing interpersonal problems, and/ or having difficulty focusing on their schoolwork. Advertisements also stated that the research team was studying the benefits of a program focused on improving body image and developing healthy coping skills. Potential participants completed a brief screening questionnaire through email or over the phone, and women identified as potentially meeting study inclusion criteria were asked to complete an in-person assessment. Each participant 
was screened by trained assessors for psychiatric comorbidity and a clinical eating disorder diagnosis using two semi-structured diagnostic interviews, the Structured Clinical Interview for DSMIV Axis I Disorders (First, Spitzer, Gibbon, \& Williams, 2002) and the Eating Disorder Examination (Fairburn \& Cooper, 1993).

Women were included in the current study if they were identified as meeting criteria for one of three eating disorder symptom or risk categories: clinical eating disorder, high risk for eating disorder onset, or low risk control. Women who met DSM-IV criteria for an eating disorder (i.e., anorexia nervosa, bulimia nervosa, binge eating disorder, or eating disorder not otherwise specified) based on data from the Eating Disorder Examination were characterized as being of clinical eating disorder status. The Weight Concerns Scale (Killen et al., 1994) is a 5-item self-report questionnaire with scores ranging 0 to 100 that was used to determine risk status. "High risk" status was defined as scoring 47 or higher on the Weight Concerns Scale (Killen et al., 1996), reporting being very afraid or terrified of gaining 3 pounds, or reporting that weight was more important than most things or the most important thing in the individual's life (Jacobi, Abascal, \& Taylor, 2004; Taylor et al., 2006). Women identified as "low risk control" status did not meet any of the clinical eating disorder or high risk status criteria and served as a control group for the current study.

Following the semi-structured interviews, interested and eligible women completed self-report questionnaires. The institutional review board at each of the participating sites approved the study protocol, and all participants provided informed consent. Measures were completed pre-treatment, between September, 2009 and April, 2010.

\section{Measures}

\section{Clinical Impairment Assessment 3.0 (CIA)}

The CIA 3.0 is a 16 -item, self-report questionnaire designed to measure psychosocial impairment due to eating disorder features in the past 28 days (Bohn \& Fairburn, 2008). Items are rated on a 4point Likert-like scale, ranging from 0 ("Not at all") to 3 ("A Lot"). The CIA generates three subscales to capture clinical impairment across specific domains, including personal, social, and cognitive. A $\mathrm{CIA}$ global score is calculated as a severity index (ranging from 0 to 48 ), with higher scores indicating greater severity of clinical impairment. The CIA has demonstrated high levels of internal consistency, test-retest reliability, sensitivity to change, construct validity, and discriminant validity in community and clinical samples of young women (Bohn et al., 2008; Reas et al., 2010).

\section{Eating Disorder Examination, 14th Edition Diagnostic Version (EDE 14.0)}

The EDE 14.0 is a semi-structured interview that generates eating disorder diagnoses based on DSM-IV criteria (Fairburn \& Cooper, 1993). In addition to determining eating disorder diagnoses, the EDE was administered to assess the frequency of objective binge episodes (defined as eating an unambiguously large amount of food with a sense of loss of control), subjective binge episodes (defined as experiencing a sense of loss of control in conjunction with eating a non-unambiguously large amount of food that is perceived as excessive by the participant), purging behaviors (vomiting, laxatives, diuretics), and driven exercise in the previous 3 months. The EDE has demonstrated high internal consistency, discriminative validity, concurrent validity, test-retest reliability, and sensitivity to change (Cooper, Cooper, \& Fairburn, 1989; Cooper \& Fairburn, 1987; Grilo, Masheb, Lozano-Blanco, \& Barry, 2003; Rizvi, Peterson, Crow, \& Agras, 2000; Rosen, Vara, Wendt, \& Leitenberg, 1990).

\section{Eating Disorder Examination - Questionnaire (EDE-Q)}

The EDE-Q is a 39-item, self-report version of the EDE that was used to assess eating disorder psychopathology in the previous 28 days, yielding a global score and four subscale scores (restraint, eating concern, weight concern, shape concern). EDE-Q global and subscale scores range from 0 to 6 , with higher scores indicating greater pathology. The EDE-Q has been shown to be internally consistent, temporally stable, and valid (Luce \& Crowther, 1999; Mond, Hay, Rodgers, \& Owen, 2006; Mond, Hay, Rodgers, Owen, \& Beumont, 2004; Peterson et al., 2007; Reas, Grilo, \& Masheb, 2006).

\section{Beck Depression Inventory-II (BDI-II)}

The BDI-II is a 21-item, self-report questionnaire that assesses depressive symptoms in the previous two weeks (Beck, Steer, \& Brown, 1996). The BDI is a widely-used measure of depressive symptoms in clinical and community samples, and has shown internal consistency, reliability, and validity in college student samples (Beck, Steer, \& Carbin, 1988; Sprinkle et al., 2002; Storch, Roberti, \& Roth, 2004).

\section{State-Trait Anxiety Inventory (STAI)}

The STAI consists of two, 20-item scales for measuring anxiety as an emotional state (state anxiety) and anxiety proneness as a personality trait (trait anxiety) (Spielberger, Gorsuch, \& Lushene, 1970). Only the state anxiety subscale was used in the current analyses. The STAI has been shown to have good construct validity, test-retest reliability, and sensitivity to change (Spielberger, 1989).

\section{Socio-demographic characteristics}

A self-report measure assessing participant demographic characteristics included age (years), racial/ethnic background (coded as nonHispanic White, African/African American, Asian/Asian American, Hispanic/Latino/Mexican American, Multi-ethnic, and Other), and education status of the participant's most educated caregiver (coded as Less than High School, High School Graduate, College Graduate, and Graduate Degree). Participants also reported on their history of treatment for an eating disorder (coded as present or absent).

\section{Analytic plan}

All analyses were conducted with SPSS v. 18.0 (SPSS Inc., Chicago, Illinois). Data were screened for normality, and skew and kurtosis were satisfactory on all continuous variables. Outliers were examined on continuous variables to see if they skewed reported group means. Overall variable means were compared with five percent trimmed means, and group means were also compared with five percent trimmed means. Excluding the outer five percent of data points did not significantly alter the mean or the pattern of results between groups. Because of this, all outliers were included in subsequent analyses. Relationships were considered significant when $p$ values were less than 0.05 . All tests were two-tailed.

Only high risk women $(n=332)$ were included in the analyses that investigated the CIA's reliability, factor structure, convergent validity (first component of construct validity), and criterion validity. The entire sample ( $n=543$ ) was used to examine the CIA's discriminant validity (second component of construct validity). Cronbach's alpha and item-total correlations were used to test the internal consistency of the CIA. Following procedures from prior CIA studies (Bohn et al., 2008; Reas et al., 2010), a principal components factor analysis examining solutions with varimax and oblimin rotations was used to investigate the CIA's dimensionality. To assess the CIA's convergent validity, Pearson correlation coefficients examined the relation between EDE-Q global score and subscales (restraint, eating concern, weight concern, shape concern) and CIA global scores. The CIA's discriminant validity was 
examined using a one-way analysis of variance (ANOVA). Eating disorder risk status (coded as low risk control, high risk, and clinical eating disorder) was the independent variable and CIA global score was the dependent variable. If the omnibus test was significant, a Tukey's HSD post hoc test examined group differences. Independent samples $t$-tests were used to investigate the CIA's criterion validity. Similar to procedures used in a prior study of the CIA (Becker et al., 2010), the independent variables were the presence of eating disorder symptoms in the past three months (objective binge episodes, subjective binge episodes, purging behaviors, driven exercise) and the presence of an eating disorder treatment history, and the dependent variable was the CIA global score.

Descriptive statistics were used to present normative data for the low risk control, high risk, and clinical eating disorder groups. Independent samples $t$-tests were used to compare differences in mean CIA global scores across studies. The low risk control group and high risk group were each compared to norms from two community samples characterized by low pathology (Reas et al., 2010; Welch et al., 2011). The clinical eating disorder group was compared with norms from two clinical samples (Bohn et al., 2008; Welch et al., 2011).

In contrast to general health-related quality of life measures that provide a global assessment of distress and functional impairment related to overall mental health, the CIA was designed to capture clinical impairment that is specifically due to eating disorder symptoms (Bohn et al., 2008). To ensure the validity of the instrument, it is important to confirm that the CIA captures the severity of clinical impairment related to an individual's eating disorder features above and beyond other forms of general psychopathology, distress, or problems s/he may be experiencing. Therefore, we attempted to disentangle the CIA's subjective appraisal of clinical impairment related to eating pathology from subjective reports of general psychological distress. In line with procedures from a prior study of the CIA (Becker et al., 2010), we explored the utility of including the BDI and STAI as covariates when conducting all analyses to control for subjective reports of general psychological distress. All results remained significant and the pattern of findings was unchanged when controlling for BDI and STAI scores. Therefore, an in-depth description of these results is not presented; however, a detailed summary of these findings is available upon request.

\section{Results}

Participants were college-age women $(20.6 \pm 2.0$ years $)$ who were at high risk for eating disorder onset $(n=332,61.1 \%)$, diagnosed with a clinical eating disorder $(n=118,21.7 \%)$, or at low risk ( $n=93,17.1 \%$ ). The racial/ethnic breakdown of the overall sample was $55.6 \%$ non-Hispanic White $(n=305), 21.6 \%$ Asian/Asian American $(n=119), 8.7 \%$ African/African American $(n=48), 8.4 \%$ Hispanic/ Latino/Mexican American $(n=46)$, 3.3\% Multi-ethnic $(n=18)$, and $2.4 \%$ Other $(n=13)$. Parents' highest level of education was a graduate degree for $46.1 \%(n=253)$, a college degree for $24.7 \%(n=136)$, a high school degree for $27.0 \%(n=148)$, and less than high school for $2.2 \%(n=12)$. Approximately $3.6 \%(n=20)$ of women reported that they previously received treatment for an eating disorder. Table 1 presents demographic data separated by risk group.

\section{Internal consistency and factor structure}

Among high risk women, the CIA demonstrated high internal consistency (Cronbach's $\alpha=0.93$ ), and item-total correlations were significantly and positively associated with the total score ( $r s$ range $=0.57$ to $0.81, p s<0.001)$.
Table 1

Participant characteristics for women at low risk for eating disorder onset, at high risk for eating disorder onset, and with a clinical eating disorder.

\begin{tabular}{lccc}
\hline & $\begin{array}{c}\text { Low risk } \\
(n=118)\end{array}$ & $\begin{array}{c}\text { High risk } \\
(n=332)\end{array}$ & $\begin{array}{l}\text { Clinical eating } \\
\text { disorder }(n=93)\end{array}$ \\
\hline Age (years) & $20.4 \pm 1.9$ & $20.6 \pm 1.9$ & $20.7 \pm 2.1$ \\
Racial/ethnic breakdown (\%) & & & \\
Non-Hispanic White & 55.8 & 56.4 & 53.5 \\
African American & 6.5 & 9.9 & 7.6 \\
Asian American & 30.1 & 18.4 & 22.9 \\
Hispanic/Latino/Mexican & 3.2 & 8.7 & 11.0 \\
Multi-ethnic & 2.2 & 3.3 & 4.2 \\
Other & 2.2 & 3.3 & 0.8 \\
Highest level of parental & & & \\
$\quad$ education (\%) & & & 3.4 \\
Less than high school & 0.0 & 2.1 & 25.4 \\
High school graduate & 26.9 & 27.1 & 21.2 \\
College graduate & 24.7 & 26.2 & 50.0 \\
Graduate degree & 48.4 & 44.3 & 8.5 \\
Previous treatment for & 0.0 & 3.0 & \\
$\quad$ an eating disorder (\%) & & & \\
\hline a $p<0.05$. & &
\end{tabular}

Among high risk women, the 16 items of the CIA fell into three components with eigenvalues exceeding 1 , which explained a total of $68.3 \%$ of the variance. Eigenvalues and factor loadings for the varimax rotation are presented in Table 2. Varimax and oblimin rotations yielded similar results. The original factor structure was retained, indicating the presence of three subscales (personal impairment, cognitive impairment, and social impairment). Cronbach's alphas for the three subscales were $0.92,0.85$, and 0.86 for the personal, cognitive, and social impairment subscales, respectively, indicating high internal consistency.

\section{Construct validity}

\section{Convergent validity}

Among high risk women, CIA global scores were significantly and positively correlated with EDE-Q global scores $(r=0.70 ; p<0.001)$ and the four EDE-Q subscales (all $p s<0.001$; restraint: $r=0.27$; eating concern: $r=0.68$; shape concern: $r=0.66$; weight concern: $r=0.65$ ). When controlling for BDI and STAI scores, the relationship between eating disorder psychopathology and CIA global scores remained significant for all analyses. Moreover, the magnitude of change in the correlation coefficients was small when controlling for these covariates, ranging from decreases between 0.01 and 0.09 .

\section{Discriminant validity}

CIA scores differed by eating disorder risk status, $F(2$, $538)=74.5 ; p<0.001$. Tukey HSD post hoc tests revealed significant differences among all three eating disorder risk groups. Consistent with the level of eating disorder risk, mean CIA global scores were highest among women with a clinical eating disorder $(17.8 \pm 10.6)$ followed by women at high risk for eating disorder onset $(10.6 \pm 8.5)$ and women at low risk (3.0 \pm 3.3$)$, respectively (Fig. 1). The results remained significant after accounting for general psychological distress (BDI and STAI scores) and the means were not significantly altered.

\section{Criterion validity}

Fig. 2 depicts the results of analyses examining criterion validity in the high risk sample. CIA global scores were significantly higher among those who reported the presence of objective binge episodes in the past three months $(14.6 \pm 10.0)$ compared to those who did not $(9.6 \pm 7.8), t(89)=-3.8, p<0.001$. Similarly, CIA global scores were significantly greater in high risk women reporting the 
Table 2

Pattern matrix for principal components analysis with varimax rotation of the three factor structure solution of Clinical Impairment Assessment (CIA) items.

\begin{tabular}{|c|c|c|c|}
\hline & $\begin{array}{l}\text { Component } 1 \text { (personal } \\
\text { impairment) }\end{array}$ & $\begin{array}{l}\text { Component } 2 \text { (cognitive } \\
\text { impairment) }\end{array}$ & $\begin{array}{l}\text { Component } 3 \text { (social } \\
\text { impairment) }\end{array}$ \\
\hline Eigenvalues & 7.87 & 1.91 & 1.15 \\
\hline$\%$ of variance explained & 49.16 & 11.93 & 7.19 \\
\hline \multicolumn{4}{|l|}{ Item } \\
\hline \multicolumn{4}{|c|}{ Over the past month, to what extent have your eating habits, exercising, or feelings about your eating, shape, or weight... } \\
\hline ...made you feel ashamed of yourself? & 0.85 & & \\
\hline ...made you upset? & 0.83 & & \\
\hline ...made you feel guilty? & 0.83 & & \\
\hline ...made you feel critical of yourself? & 0.81 & & \\
\hline ...made you worry? & 0.74 & & \\
\hline ...made you feel like a failure? & 0.71 & & \\
\hline ...made you forgetful? & & 0.84 & \\
\hline ...made you absent-minded? & & 0.81 & \\
\hline ...affected your work performance (if applicable)? & & 0.71 & \\
\hline ...affected your ability to make everyday decisions? & & 0.65 & \\
\hline ...made it difficult to concentrate? & & 0.58 & \\
\hline ...interfered with you doing things you used to enjoy? & & & 0.50 \\
\hline ...interfered with meals with family or friends? & & & 0.79 \\
\hline ...stopped your from going out with others? & & & 0.79 \\
\hline ...made it difficult to eat out with others? & & & 0.77 \\
\hline ...interfered with your relationships with others? & & & 0.59 \\
\hline Mean \pm SD & $6.8 \pm 4.8$ & $2.6 \pm 2.8$ & $2.3 \pm 3.0$ \\
\hline Median (range) & $6(0-18)$ & $2(0-17)$ & $1(0-15)$ \\
\hline
\end{tabular}

presence of subjective binge episodes in the previous three months $(14.5 \pm 9.0)$ than high risk women without subjective binge episodes $(8.4 \pm 7.4), t(219)=-6.4, p<0.001$. CIA global scores were also significantly higher among those reporting the presence of purging behaviors in the past three months $(16.3 \pm 11.1)$ compared to those who did not $(10.2 \pm 8.1), t(27)=-2.8, p=0.01$. As compared to those without driven exercise (10.4 \pm 8.5 ), high risk women reporting driven exercise in the previous three months had significantly higher CIA global scores $(14.6 \pm 8.4), t(329)=-2.0$, $p=0.05$. Finally, high risk women with a history of eating disorder treatment reported significantly greater CIA scores $(18.9 \pm 12.2)$ than those without a prior treatment history $(10.4 \pm 8.3)$, $t(9)=-2.1, p=0.05$. The results remained significant after accounting for general psychological distress (BDI and STAI scores) and the means were not significantly altered.

\section{Normative data}

Table 3 presents the means and standard deviations, as well as corresponding percentile rank values, for the CIA global score in the low risk control, high risk, and clinical eating disorder groups. The low risk control group in the current study had significantly lower

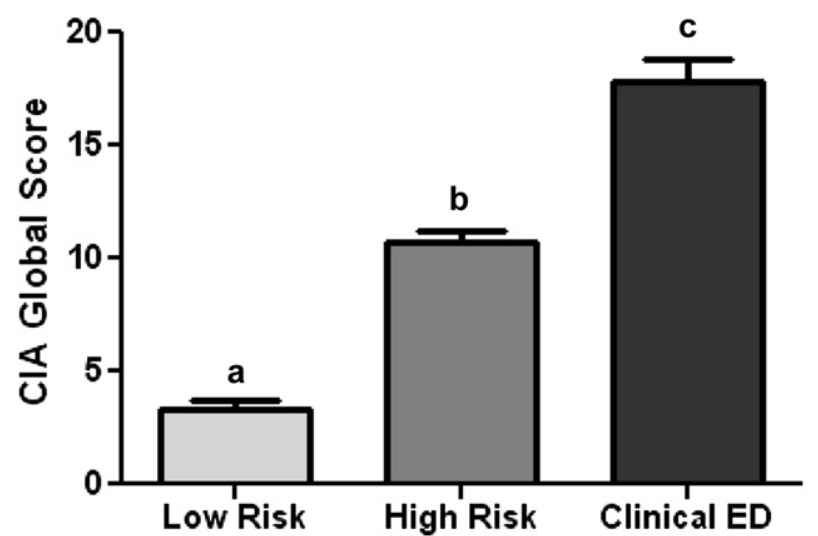

Fig. 1. Discriminant validity of the Clinical Impairment Assessment (CIA): Comparison of CIA global scores among low risk controls $(n=118)$, high risk women $(n=332)$, and women with a clinical eating disorder (ED; $n=93$ ). Note: Different letters represent significant differences between groups at the $p<0.05$ level. mean CIA global scores $(3.0 \pm 3.3)$ than community samples from Norway $(6.4 \pm 7.5), t(554)=4.4, p<0.001$, and from Sweden $(8.3 \pm 9.4), t(219)=5.7, p<0.001$. The high risk group reported significantly higher mean CIA global scores than the Norwegian sample, $t(768)=7.4, p<0.001$, and the Swedish sample, $t(1090)=4.1, p<0.001$. The clinical eating disorder group in the current study had significantly lower mean CIA global scores $(17.8 \pm 10.6)$ as compared to clinical samples from the United Kingdom $(31.2 \pm 9.9), t(214)=9.6, p<0.001$, and Sweden $(30.2 \pm 10.2), t(2474)=11.5, p<0.001$.

\section{Discussion}

The Clinical Impairment Assessment (CIA) was developed to capture impairment in psychosocial domains among individuals with varying levels of eating disorder symptom severity (Bohn \& Fairburn, 2008). The current study was the first to examine the psychometric properties of the CIA among a sample of women at high risk for developing an eating disorder. Results indicate that the

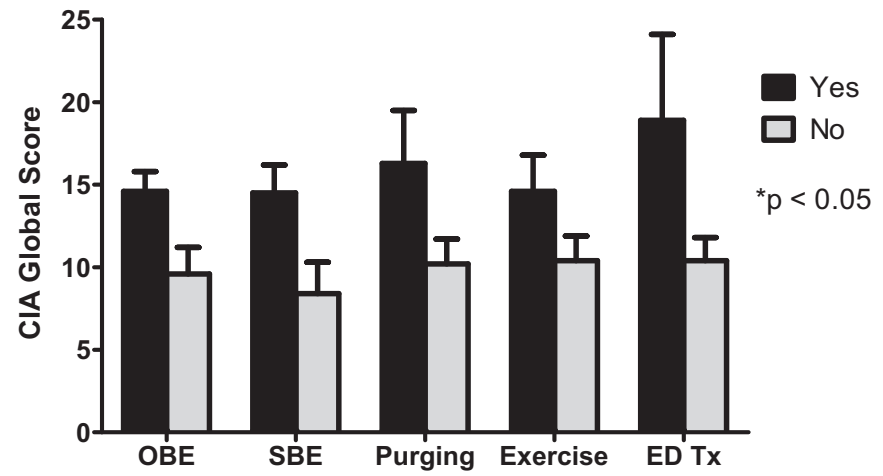

Fig. 2. Criterion validity of the Clinical Impairment Assessment (CIA) in women at high risk for eating disorder onset: comparison of CIA global scores among high risk women with and without eating disorder (ED) symptoms. Note: The presence of the following symptoms in the three months prior to assessment were assessing with the Eating Disorder Examination: OBE = Objective binge episodes, SBE = Subjective binge episodes, Purging $=$ Purging behaviors (vomiting, laxative use, diuretic use), and Exercise $=$ Driven exercise. Participants also reported on their lifetime history of any treatment for an eating disorder (ED Tx). 
Table 3

Normative data for Clinical Impairment Assessment (CIA) global scores in collegeage women at low risk for an eating disorder, at high risk for eating disorder onset, and with a clinical eating disorder.

\begin{tabular}{lcll}
\hline & $\begin{array}{l}\text { Low risk } \\
(n=118)\end{array}$ & $\begin{array}{l}\text { High risk } \\
(n=332)\end{array}$ & $\begin{array}{l}\text { Clinical eating } \\
\text { disorder }(n=93)\end{array}$ \\
\hline $\begin{array}{l}\text { Mean } \pm \text { SD } \\
\text { Percentile rank }\end{array}$ & $3.3 \pm 3.6$ & $10.7 \pm 8.5$ & $17.8 \pm 10.6$ \\
5 & 0.0 & 0.0 & 3.0 \\
10 & 0.0 & 1.0 & 4.0 \\
25 & 0.0 & 4.0 & 10.0 \\
50 & 2.0 & 9.0 & 16.0 \\
75 & 5.0 & 16.0 & 25.0 \\
90 & 9.0 & 23.0 & 32.0 \\
95 & 11.0 & 28.0 & 38.0 \\
100 & 16.0 & 43.0 & 48.0 \\
\hline
\end{tabular}

CIA demonstrated strong psychometric properties within our sample of high risk women and revealed meaningful patterns of clinical impairment among clinical eating disorder, high risk, and low risk control women. Thus, our data indicate that the CIA is a reliable and valid tool for assessing the pervasive impact of eating disorder symptoms on functioning across important domains in college-age women.

Findings suggest that the CIA is a reliable measure among high risk women, as it demonstrated high internal consistency (Cronbach's $\alpha=0.93$ ) and item-total correlation values were moderate to strong ( $r$ range $=0.56-0.81$ ). Among this high risk sample, the CIA measured a cohesive construct and all individual items appeared to contribute to the CIA global score-the designated index of impairment severity (Bohn et al., 2008). These results are comparable to the reliability testing in prior studies of the CIA with community and clinical samples (Bohn et al., 2008; Reas et al., 2010). The three factor structure of the CIA, which includes personal, cognitive, and social impairment subscales, that was previously described (Bohn et al., 2008; Reas et al., 2010) was replicated in the current study. Each of the subscales demonstrated high internal consistency (Cronbach's $\alpha=0.85-0.92$ ). These findings support the use of both global and domain-specific CIA scores to assess impairment related to eating disorder features. Overall, findings from the current study and prior work support the reliability and factor structure of the CIA in samples characterized by low eating pathology, disordered eating pathology that places individuals at high risk for eating disorder onset, and full syndrome eating disorders.

The CIA demonstrated good construct validity in the current study. Self-reported eating pathology, as assessed by the global and subscale scores of the Eating Disorder Examination Questionnaire (EDE-Q), was positively correlated with global CIA scores among high risk women. These findings are consistent with prior studies of the CIA in eating disorder samples (Becker et al., 2010; Bohn et al., 2008) and in women with low pathology from the general population (Reas et al., 2010). The CIA also discriminated between women across the spectrum of eating disorder risk. Women with clinical eating disorders reported the highest CIA global scores, while women in the low risk control group reported the lowest CIA global scores. As expected, women at high risk for eating disorder onset reported significantly less clinical impairment than women with a clinical eating disorder but significantly more impairment than women with very low pathology. In general, the CIA appears to differentiate among women across the spectrum of disordered eating, lending strength to the clinical utility of the instrument. Findings also highlight that it may be possible to more clearly operationalize the construct of clinical impairment for mental disorders, which is currently somewhat ambiguous, using a brief self-report measure similar to the structure of the CIA.
Findings indicated that the CIA has good criterion validity with indices of eating disorder psychopathology among women at high risk for eating disorder onset. The presence of objective binge episodes was associated with significantly higher CIA global scores than the absence of objective binge episodes, corresponding to the only prior examination of criterion validity of the CIA that took place in Fijian adolescent schoolgirls (Becker et al., 2010). Notably, the severity of clinical impairment, as assessed by CIA global scores, was comparable between the presence of objective and subjective binge episodes. These data imply that the presence of a binge episode regardless of size may be clinically meaningful-at least among this high risk sample-which supports emerging research indicating that the experience of loss of control may be more salient than the size of a binge episode (Wolfe, Wood Baker, Smith, \& KellyWeeder, 2009). Further, high risk women reporting purging behaviors had significantly greater CIA global scores than those without any purging behaviors. This differs from Becker et al.'s (2010) study in which adolescent females who endorsed vomiting or laxative use did not significantly differ on CIA global scores from those without these purging behaviors. It is possible that purging behaviors are simply not indicative of impairment in Fijian cultures because they may be more socially acceptable, whereas purging behaviors are good markers of impairment in the United States because they are culturally associated with more social distress and shame. Key differences between samples, including age or the overall severity of eating pathology, may also account for these inconsistent findings. Finally, high risk women reporting driven exercise-a key non-purging behavior-had higher CIA global scores. Overall, the CIA appears to capture clinical impairment related to a broad range of indices of eating disorder psychopathology among this high risk sample. These findings also highlight the importance of examining clinical impairment in relation to indices of psychopathology across cultures, as this may have important implications for the universality of current diagnostic criteria that requires individuals to experience distress or functional impairment related to their mental disorder symptoms.

In our examination of the CIA's criterion validity, results indicate that high risk women reporting a history of eating disorder treatment had clinically significant impairment related to their symptoms. High risk women with an eating disorder treatment history reported a mean CIA global score of approximately 19, which is comparable to prior data suggesting that a CIA global score of 16 is indicative of a full syndrome eating disorder case (Bohn et al., 2008) and our current mean CIA global score of 18 among women with eating disorders. Since high risk status in the current study was defined by the presence of elevated weight and shape concerns, it is possible that disordered eating attitudes continued to linger despite previous treatment or that this eating pathology resurfaced following treatment cessation. This residual eating pathology or clinical impairment may place these women with a previous treatment history at an especially high risk for relapse. Consistent with this notion, research suggests that the presence of elevated weight and shape concerns following the cessation of treatment for an eating disorder is a risk factor for relapse into a full syndrome eating disorder (Carter, Blackmore, Sutandar-Pinnock, \& Woodside, 2004; Channon \& deSilva, 1985; Keel, Dorer, Franko, Jackson, \& Herzog, 2005; McFarlane, Olmsted, \& Trottier, 2008). Prospective data also indicate that psychosocial dysfunction following symptom remission was a predictor of relapse among individuals with bulimia nervosa (Keel et al., 2005). The characterization of the role of clinical impairment in the risk for relapse from an eating disorder is sorely needed; it may be also be worthwhile to investigate whether the notion of "recovery" from an eating disorder may need to include a greater emphasis on level of clinical impairment. The CIA appears to be a useful tool to assess impairment in such studies. 
Additionally, findings point to the potential importance of incorporating relapse prevention strategies into routine clinical practice.

In addition to examining the psychometric properties of the CIA among a high risk sample, we also sought to provide the first normative data for the CIA in non-treatment seeking, college-age women with varying levels of eating disorder psychopathology from the United States. Women with a clinical eating disorder had an average CIA global score of approximately 18 . This clinical norm was significantly lower than the clinical samples of young Swedish women and individuals from the United Kingdom (CIA global scores of 30) (Bohn et al., 2008; Welch et al., 2011), but appears similar to the recommended clinical cut-off score of 16 (Bohn et al., 2008). Women at high risk for developing an eating disorder reported a mean CIA global score of approximately 11 , which was significantly higher than norms from community samples of young women characterized by low pathology (Reas et al., 2010; Welch et al., 2011). This study provides the first account of the severity of impairment among a high risk, college-age population, which may be greater than the general population. Finally, women with very low pathology in the current study had an average CIA global score of 3 , which was significantly lower than prior studies finding mean CIA global scores between 6 and 8 in the general population (Reas et al., 2010; Welch et al., 2011). However, it is likely that these general population samples also included women at high risk for eating disorder onset or with significant subclinical pathology, accounting for these higher norms.

Some caution should be exerted when interpreting differences in scores and when generalizing the norms from the current study to the general United States population. Our norms represent a college-age population of women who self-selected for the study based on body image concerns, interpersonal problems, or concentration difficulties in school. However, these issues are common among college-age women and so a broad base of the population was likely reached. It is also noteworthy that women were recruited from a diverse array of schools-ranging from community colleges to private universities-and represented a wide range of socioeconomic status and racial/ethnic backgrounds from two areas of the United States. Moreover, scores on the Weight Concerns Scale (Killen et al., 1994) — the study's assessment of body image concerns-were normally distributed across the nearly entire range of potential scores ( 0 to 95 out of a possible 0 to 100 range) and the sample mean and median $(M=54.1$; median $=56)$ was very close to the center of the distribution of the range. Therefore, our norms are likely representative of the college-age women, but large-scale, epidemiological studies in the United States are needed to make this determination. More work is needed in culturally- and demographically-diverse settings to replicate these findings.

A primary strength of the current study is the large sample of young women at high risk for an eating disorder by virtue of having elevated weight and shape concerns, which captures a critical subthreshold group. The study's attempts to disentangle women's subjective appraisals of clinical impairment related to eating pathology from subjective reports of general psychological distress also represents a significant strength. Overall, these results suggest that the CIA is sensitive to differences in self-evaluations of clinical impairment due to eating disorder symptoms independent of subjective reports of general psychological distress. These analyses allow us to feel more confident that the CIA is assessing impairment that is the direct result of eating disorder features-a critical criterion for defining a mental disorder in DSM-5 (Stein et al., 2010). The current study is also the first study of the CIA in a sample from the United States and provides needed normative data. The inclusion of clinical eating disorder and low risk groups is another key strength because it allowed the study to capture clinical impairment across a individuals with varied levels of pathology and to examine the discriminant validity of the CIA with an adequate sample size. Our sample was also more ethnically diverse compared to most prior CIA studies in women (Bohn et al., 2008; Reas et al., 2010; Welch et al., 2011), which occurred primarily in Caucasians.

Limitations of the current study include the limited age range of the sample (most individuals were between 18 and 22 years) and that it was comprised entirely of women. However, earlier work primarily examined the CIA among young women as well, which allowed us to more easily interpret results across studies during these early psychometric studies of the CIA. Further investigation of the CIA among racially/ethnically diverse populations, men, and individuals spanning a broad age range is required to increase the generalizability of findings related to the CIA's clinical utility. Additionally, the current study did not use objective measures of clinical impairment, such as expert clinician ratings or collateral reports, to provide a more stringent test of construct validity. More research is needed to examine the association between the CIA and blinded, external ratings of clinical impairment among individuals who endorse a range of disordered eating attitudes and behaviors. Finally, the cross-sectional nature of the study design limits our ability to make causal inferences about the relation between the development of eating disorder symptoms and clinical impairment. The CIA, however, does attempt to capture causality by asking participants to rate the extent to which they are impaired because of the experience of eating pathology. It is critical for future studies to examine the performance of the CIA prospectively and throughout the course of treatment to investigate its predictive validity and utility as an outcome measure.

Overall, the current study indicates that the CIA is a psychometrically strong measure among young women at high risk for eating disorder onset and is useful for differentiating subgroups of individuals with varying levels of eating disorder psychopathology. Our results provide the first set of norms for the CIA in a United States sample of college-age women. Clinical impairment, as measured by the CIA, should be considered an important construct of clinical research, diagnostic decision-making, and treatment assessment over time. Given the promising findings associated with the CIA and the increasing importance of clinical impairment recommended for inclusion in the forthcoming DSM-5 (APA, 2010; Stein et al., 2010), future studies should consider evaluating clinical impairment instruments similar to the CIA across a broader range of mental disorders.

\section{Funding}

R01 MH081125 (C.B. Taylor and D.E. Wilfley); K24 070446 (D.E. Wilfley); T32 HL007456.

\section{References}

Ackard, D. M., Fulkerson, J. A., \& Neumark-Sztainer, D. (2011). Psychological and behavioral risk profiles as they relate to eating disorder diagnoses and symptomatology among a school-based sample of youth. International Journal of Eating Disorders, 44(5), 440-446.

American Psychiatric Association (APA). (2000). Diagnostic and statistical manual of mental disorders (Revised 4th ed.). Arlington, VA: American Psychiatric Association.

American Psychiatric Association (APA). (2010). Diagnostic and Statistical Manual of Mental Disorders (DSM-5): The future of psychiatric diagnosis. Retrieved January 20, 2012, 2011, from. http://www.dsm5.org.

Beck, A. T., Steer, R. A., \& Brown, G. K. (1996). Manual for the Beck Depression Inventory-II. San Antonio, TX: Psychological Corporation.

Beck, A. T., Steer, R. A., \& Carbin, M. G. (1988). Psychometric properties of the Beck Depression Inventory: twenty-five years of evaluation. Clinical Psychology Review, 8(1), 77-100.

Becker, A. E., Thomas, J. J., Bainivualiku, A., Richards, L., Navara, K., Roberts, A. L. et al. (2010). Adaptation and evaluation of the Clinical Impairment Assessment to assess disordered eating related distress in an adolescent female ethnic Fijian population. International Journal of Eating Disorders, 43(2), 179-186. 
Bohn, K., Doll, H. A., Cooper, Z., O'Connor, M., Palmer, R. L., \& Fairburn, C. G. (2008). The measurement of impairment due to eating disorder psychopathology. Behavior Research and Therapy, 46(10), 1105-1110.

Bohn, K., \& Fairburn, C. G. (2008). Clinical impairment questionnaire (CIA 3.0). In C. G. Fairburn (Ed.), Cognitive behavior therapy and eating disorders (pp. 315-317). New York: Guilford Press.

Carter, J. C., Blackmore, E., Sutandar-Pinnock, K., \& Woodside, D. B. (2004). Relapse in anorexia nervosa: a survival analysis. Psychological Medicine, 34(4), 671-679.

Channon, S., \& deSilva, W. P. (1985). Psychological correlates of weight gain in patients with anorexia nervosa. Journal of Psychiatry Research, 19(2-3), 267-271.

Cooper, Z., Cooper, P. J., \& Fairburn, C. G. (1989). The validity of the eating disorder examination and its subscales. British Journal of Psychiatry, 154, 807-812.

Cooper, Z., \& Fairburn, C. G. (1987). The eating disorder examination: a semistructured interview for the assessment of the specific psychopathology of eating disorders. International Journal of Eating Disorders, 6, 1-8.

Fairburn, C. G. (2008). Cognitive behavior therapy and eating disorders. New York: Guilford Press.

Fairburn, C. G., \& Cooper, Z. (1993). The eating disorder examination. In (12th ed.). In Fairburn, C. G., \& Wilson, G. T. (Eds.), Binge eating: Nature, assessment and treatment, Vol. 317-60 New York: Guilford.

First, M. B., Spitzer, R. L., Gibbon, M., \& Williams, J. B. W. (2002). Structured Clinical Interview for DSM-IV-TR Axis I Disorders, Research Version. New York: Biometrics Research, New York State Psychiatric Institute.

Grilo, C. M., Masheb, R. M., Lozano-Blanco, C., \& Barry, D. T. (2003). Reliability of the eating disorder examination in patients with binge eating disorder. International Journal of Eating Disorders, 35(1), 80-85.

Hudson, J. I., Hiripi, E., Pope, H. G., \& Kessler, R. C. (2007). The prevalence and correlates of eating disorders in the National Comorbidity Survey Replication. Biological Psychiatry, 61(3), 348-358.

Jacobi, C., Abascal, C., \& Taylor, C. B. (2004). Screening for eating disorders and highrisk behavior: caution. International Journal of Eating Disorders, 36(3), 280-285.

Jacobi, C., Fittig, E., Bryson, S. W., Wilfley, D. E., Kraemer, H. C., \& Taylor, C. B. (2011) Who is really at risk? Identifying risk factors for subthreshold and full syndrome eating disorders in a high-risk sample. Psychological Medicine, (Epub ahead of print).

Keel, P. K., Dorer, D. J., Franko, D. L., Jackson, S. C., \& Herzog, D. B. (2005). Postremission predictors of relapse in women with eating disorders. American Journal of Psychiatry, 162(12), 2263-2268.

Killen, J. D., Taylor, C. B., Hayward, C., Haydel, K. F., Wilson, D. M., Hammer, L., et al. (1996). Weight concerns influence the development of eating disorders: a 4year prospective study. Journal of Consulting and Clinical Psychology, 64(5), 936-940.

Killen, J. D., Taylor, C. B., Hayward, C., Wilson, D. M., Haydel, K. F., Hammer, L. D., et al. (1994). Pursuit of thinness and onset of eating disorder symptoms in a community sample of adolescent girls: a three-year prospective analysis. International Journal of Eating Disorders, 16(3), 227-238.

Luce, K. H., \& Crowther, J. H. (1999). The reliability of the eating disorder examination-self-report questionnaire (EDE-Q). International Journal of Eating Disorders, 25(3), 349-351.

McFarlane, T., Olmsted, M. P., \& Trottier, K. (2008). Timing and prediction of relapse in a transdiagnostic eating disorder sample. International Journal of Eating Disorders, 41(7), 587-593.
Mond, J. M., Hay, P. J., Rodgers, B., \& Owen, C. (2006). Eating disorder examination questionnaire (EDE-Q): norms for young adult women. Behavior Research and Therapy, 44(1), 53-62.

Mond, J. M., Hay, P. J., Rodgers, B., Owen, C., \& Beumont, P. J. V. (2004). Validity of the eating disorder examination questionnaire (EDE-Q) in screening for eating disorders in community samples. Behavior Research and Therapy, 42(5), 551-567.

Peebles, R., Hardy, K. K., Wilson, J. L., \& Lock, J. D. (2010). Are diagnostic criteria for eating disorders markers of medical severity? Pediatrics, 125(5), e1193-1201.

Peterson, C. B., Crosby, R. D., Wonderlich, S. A., Joiner, T., Crow, S. J., Mitchell, J. E. et al. (2007). Psychometric properties of the eating disorder examinationquestionnaire: factor structure and internal consistency. International Journal of Eating Disorders, 40(4), 386-389.

Polivy, J., \& Herman, C. P. (2002). Causes of eating disorders. Annual Review of Psychology, 53, 187-213.

Reas, D. L., Grilo, C. M., \& Masheb, R. M. (2006). Reliability of the eating disorder examination-questionnaire in patients with binge eating disorder. Behavior Research and Therapy, 44(1), 43-51.

Reas, D. L., Ro, O., Kapstad, H., \& Lask, B. (2010). Psychometric properties of the Clinical Impairment Assessment: norms for young adult women. International Journal of Eating Disorders, 43, 72-76.

Rizvi, S. L., Peterson, C. B., Crow, S. J., \& Agras, W. S. (2000). Test-retest reliability of the eating disorder examination. International Journal of Eating Disorders, 28(3), $311-316$.

Rosen, J. C., Vara, L., Wendt, S., \& Leitenberg, H. (1990). Validity studies of the eating disorder examination. International Journal of Eating Disorders, 9, 519-528.

Spielberger, C. D. (1989). State-Trait Anxiety Inventory: A comprehensive bibliography, Vol. 2. Palo Alto, CA: Consulting Psychologists Press.

Spielberger, C. D., Gorsuch, R. L., \& Lushene, R. E. (1970). Manual for the State-Trait Anxiety Inventory. Palo Alto, CA: Consulting Psychologists Press.

Sprinkle, S. D., Lurie, D., Insko, S. L. Atkinson, G., Jones, G. L., Logan, A. R., et al. (2002). Criterion validity, severity cut scores, and test-retest reliability of the Beck Depression Inventory-II in a university counseling center sample. Journa of Counseling Psychology, 49(3), 381-385.

Stein, D. J., Phillips, K. A., Bolton, D., Fulford, K. W. Sadler, J. Z, \& Kendler, K. S. (2010). What is a mental/psychiatric disorder? From DSM-IV to DSM-5. Psychological Medicine, 40, 1759-1765.

Storch, E. A., Roberti, J. W., \& Roth, D. A. (2004). Factor structure, concurrent validity, and internal consistency of the beck depression inventory-second edition in a sample of college students. Depression and Anxiety, 19(3), 187-189.

Swanson, S. A., Crow, S. J., Le Grange, D., Swendsen, J., \& Merikangas, K. R. (2011) Prevalence and correlates of eating disorders in adolescents: results from the national comorbidity survey replication adolescent supplement. Archives of General Psychiatry, 68(7), 714-723.

Taylor, C. B., Bryson, S., Luce, K. H., Cunning, D., Celio-Doyle, A., Abascal, L. B., et al. (2006). Prevention of eating disorders in at-risk college-age women. Archives of General Psychiatry, 63, 881-888.

Welch, E., Birgegard, A., Parling, T., \& Ghaderi, A. (2011). Eating disorder examination questionnaire and clinical impairment assessment questionnaire: general population and clinical norms for young adult women in Sweden. Behavior Research and Therapy, 49, 85-91.

Wolfe, B. E., Wood Baker, C., Smith, A. T., \& Kelly-Weeder, S. (2009). Validity and utility of the current definitions of binge eating. International Journal of Eating Disorders, 42, 674-686. 